\title{
MicroRNA-33 regulates the NLRP3 inflammasome signaling pathway in macrophages
}

\author{
QINGYUN XIE $^{1 *}$, MENG WEI $^{2 *}$, BO ZHANG ${ }^{1}$, XIA KANG $^{1}$, DA LIU $^{1}$, WEI ZHENG $^{1}$, \\ XIANMING PAN $^{1}$, YI QUAN ${ }^{1}$, DONGFA LIAO ${ }^{1}$ and JUN SHEN ${ }^{1}$ \\ Departments of ${ }^{1}$ Orthopedics and ${ }^{2}$ Nephrology and Rheumatology, \\ Chengdu Military General Hospital, Jinniu, Chengdu, Sichuan 610083, P.R. China
}

Received June 13, 2016; Accepted May 11, 2017

DOI: $10.3892 / \mathrm{mmr} .2017 .8224$

\begin{abstract}
The nucleotide binding domain and leucine-rich repeat pyrin 3 domain (NLRP3) inflammasome/interleukin (IL)-1 $\beta$ axis serves an essential role in regulating the development of rheumatoid arthritis (RA). The dysregulation of cellular metabolism, such as mitochondrial dysfunction, results in the activation of the NLRP3 inflammasome. microRNA (miR)-33 has previously been identified to be a regulator of lipid metabolism and mitochondrial function. However, whether miR-33 regulates the NLRP3 inflammasome/IL-1 $\beta$ axis remains unknown. In the present study, it was observed that an miR-33 mimic or anti-miR-33 markedly stimulated or inhibited, respectively, IL-1 $\beta$ protein expression levels in mouse peritoneal macrophages. Mechanistically, miR-33 upregulated the expression of NLRP3 mRNA and protein as well as caspase-1 activity in primary macrophages. In addition, the results demonstrated that miR-33 impaired mitochondrial oxygen consumption rates, resulting in the accumulation of cellular reactive oxygen species, which stimulated NLRP3 expression, caspase- 1 activity and IL- $1 \beta$ secretion. The results of the present study demonstrated that miR-33 levels and NLRP3 inflammasome activity were increased in peripheral blood monocytes from patients with RA patients compared with healthy donors. In conclusion, the present study identified miR-33 to be a positive regulator of the NLRP3 inflammasome
\end{abstract}

Correspondence to: Dr Meng Wei, Department of Nephrology and Rheumatology, Chengdu Military General Hospital, 270 Rongdu Avenue, Jinniu, Chengdu, Sichuan 610083, P.R. China

E-mail: mengweicd@sina.com

*Contributed equally

Abbreviations: miR, microRNA; RA, rheumatoid arthritis; NLRP3, nucleotide binding domain and leucine-rich repeat pyrin 3 domain; IL-1 $\beta$, interleukin-1 $\beta$; OCR, oxygen consumption rate; ROS, reactive oxygen species

Key words: microRNA-33, interleukin-1 $\beta$, mitochondrial function, rheumatoid arthritis, inflammasome in macrophages. The miR-33/NLRP3 inflammasome pathway may therefore be involved in RA development.

\section{Introduction}

Inflammasomes are cytosolic multiprotein complexes which induce the production of proinflammatory cytokines, primarily interleukin (IL)- $1 \beta$ and IL-18, in response to pathogens or danger signals $(1,2)$. Inflammasome complexes are composed primarily of the apoptosis-associated speck-like protein containing a caspase activation and recruitment domain, a sensor protein and caspase-1. Distinct inflammasome complexes specifically respond to various signals via a diverse set of associated sensor proteins. The nucleotide binding domain and leucine-rich repeat pyrin 3 domain (NLRP3) is a well-characterized sensor protein that forms the NLRP3 inflammasome, which is activated by exogenous pathogens or endogenous danger signals, including mitochondrial dysfunction and the accumulation of reactive oxygen species (ROS) (3-5). Activation of the NLRP3 inflammasome leads to the auto-activation of procaspase-1; procaspase-1 subsequently catalyzes pro-IL-1 $\beta$ maturation to IL- $1 \beta$, which is then secreted (1-5).

Rheumatoid arthritis (RA) is a chronic inflammatory disease, which is induced by genetic and/or environmental factors. Of these, pro-inflammatory cytokines, such as tumor necrosis factor- $\alpha$ and IL-1 $\beta$, are well-characterized inducers (6-8). Notably, the NLRP3 inflammasome is reportedly associated with RA susceptibility and severity in certain animal models and human studies (9-11). A previous study involving a spontaneous arthritis mouse model (A20 ${ }^{\text {mye-KO }}$ mice) demonstrated that the pathology of arthritis may be associated with the NLRP3 inflammasome/IL-1 $\beta$ axis (12).

Cellular metabolism has been increasingly recognized as an endogenous controller of NLRP3 inflammasome activation $(3,5)$. Saturated free fatty acids are potent simulators of the NLRP3 inflammasome and IL-1 $\beta$ secretion (13). In addition, mitochondrial dysfunction and ROS accumulation may induce NLRP3 expression and inflammasome activation $(4,5)$. Modulation of cellular metabolism has been demonstrated to regulate NLRP3 inflammasome activity and associated disease pathologies, such as Alzheimer's disease and liver fibrosis, in a number of previous studies $(1,14,15)$. 
MicroRNAs (miRs) form one of the largest groups of post-transcriptional regulatory factors $(16,17)$. miRs possess 2-8 bases at the 5'-terminal that bind to the 3'-untranslated region (3'-UTR) of a target mRNA, thereby repressing gene translation and decreasing protein expression (17). By regulating the expression of key enzymes or regulatory factors, miRs serve a role in regulating cellular metabolism (18). miR-33 has been previously demonstrated to regulate cellular lipid metabolism and to repress the expression of genes associated with cholesterol efflux (19), high-density lipoprotein biogenesis (20) and fatty acid oxidation (21). A recent study reported that a macrophage-specific miR-33 deletion increases oxidative respiration, enhances spare respiratory capacity and induces the expression of an M2 macrophage polarization-associated gene profile (22). However, the mechanism associating miR-33 with macrophage activation remains to be completely elucidated.

In the present study, the authors hypothesized that miR-33 may regulate the mitochondrial function-dependent activity of the NLRP3 inflammasome in macrophages. Through gain and loss of function studies, the role of miR-33 in regulating the NLRP3 inflammasome/IL-1 $\beta$ axis in primary mouse macrophages was investigated. In addition, the expression of miR-33 and NLRP3 inflammasome-associated genes was measured in peripheral blood monocytes obtained from patients with RA and compared with those of healthy donors.

\section{Materials and methods}

Isolation of peritoneal macrophages. A total of $130 \mathrm{C} 57 \mathrm{BL} / 6$ mice, provided by Model Animal Resource Information Platform of Nanjing University (Nanjing, China), were used the present study. Mice, housed five per cage, were supplied with sterile water and food ad libitum in a pathogen-free facility with a 12-h light/dark cycle. The temperature was maintained at $25^{\circ} \mathrm{C}$ and the humidity at $55 \%$. Each mouse (male; age, 8 weeks; weight, 20-24 g) was injected intraperitoneally with $2 \mathrm{ml} 3 \%$ thioglycollate (Difco; BD Biosciences, Franklin Lakes, NJ, USA) on day 1, and was sacrificed with $\mathrm{CO}_{2}$ or isoflurane on day 3. Following an intraperitoneal injection of $5 \mathrm{ml}$ Dulbecco's modified Eagle's medium (cat. no. 11965118; Gibco; Thermo Fisher Scientific, Inc., Waltham, MA, USA) containing $10 \%$ fetal bovine serum (cat. no. 10100147; Thermo Fisher Scientific, Inc.) and 1\% penicillin and streptomycin (cat. no. 15070063; Gibco; Thermo Fisher Scientific, Inc.), the peritoneal cells were collected and transferred to cell culture dishes maintained at $37^{\circ} \mathrm{C}$ in a humidified $5 \% \mathrm{CO}_{2}$ atmosphere. Following $2 \mathrm{~h}$, the floating cells were removed by washing the cells with phosphate-buffered saline (PBS). The adherent cells were considered to be peritoneal macrophages and employed for subsequent experiments. The use of animals in the present study was approved by the Ethics Committee of Chengdu Military General Hospital (Chengdu, China).

Treatment of the peritoneal macrophages. All treated macrophages were maintained at $37^{\circ} \mathrm{C}$ in a humidified $5 \% \mathrm{CO}_{2}$ atmosphere.

For measurement of mRNA and protein expression, peritoneal macrophages were transfected with PBS (control), a scramble miR (100 nM, cat. no. AM17010), $100 \mathrm{nM}$ miR-33 mimic (cat. no. MC12410) or $100 \mathrm{nM}$ anti-miR-33 (cat. no. AM12410) (all from Thermo Fisher Scientific, Inc.) for $5 \mathrm{~h}$ and then treated with PBS or lipopolysaccharide (LPS; $50 \mathrm{ng} / \mathrm{ml}$, cat. no. L2630; Sigma-Aldrich) for $24 \mathrm{~h}$ before cells were harvested. For measurement of ELISA, peritoneal macrophages were treated as aforementioned before collecting the supernatants.

For measurement of ROS levels, peritoneal macrophages were treated as aforementioned. Alternatively, peritoneal macrophages were transfected with a scramble miR $(100 \mathrm{nM})$ or an miR-33 mimic $(100 \mathrm{nM})$ for $5 \mathrm{~h}$ and subsequently treated with PBS (control), $2 \mu \mathrm{g} / \mathrm{ml}$ oligomycin, $1 \mu \mathrm{g} / \mathrm{ml}$ carbonyl cyanide $\mathrm{p}$-(trifluoromethoxy) phenylhydrazone (FCCP), $1 \mu \mathrm{g} / \mathrm{ml}$ rotenone $+1 \mu \mathrm{g} / \mathrm{ml}$ antimycin A (all the mitochondrial inhibitors were from the Agilent Seahorse XF Cell Mito Stress test kit; cat. no. 103015-100; Agilent Technologies, Inc., Santa Clara, CA, USA), 20 mM $\mathrm{N}$-acetylcysteine (NAC; cat. no. S0077; Beyotime Institute of Biotechnology, Shanghai, China) or $10 \mathrm{mM}$ glutathione (GSH; cat. no. S0073; Beyotime Institute of Biotechnology) for $24 \mathrm{~h}$. For measurement of mitochondrial oxygen consumption rate (OCR), peritoneal macrophages were transfected with a scrambled miR $(100 \mathrm{nM})$ or an miR-33 mimic $(100 \mathrm{nM})$ for $24 \mathrm{~h}$, followed by the treatment of oligomycin $(2 \mu \mathrm{g} / \mathrm{ml})$, FCCP $(1 \mu \mathrm{g} / \mathrm{ml})$ and rotenone $(1 \mu \mathrm{g} / \mathrm{ml})$ plus antimycin A $(1 \mu \mathrm{g} / \mathrm{ml})$ one after another in the XFe96 Extracellular Flux analyzer (Seahorse Bioscience; Agilent Technologies, Inc.). For measurement of caspase-1 activity, peritoneal macrophages were transfected with a scrambled miR $(100 \mathrm{nM})$ or miR-33 mimic (100 $\mathrm{nM}$ ) for $5 \mathrm{~h}$, and then treated with LPS (50 ng/ml) or PBS as a control, plus DMSO, NAC (20 mM), GSH $(10 \mathrm{mM})$ or FCCP $(1 \mu \mathrm{g} / \mathrm{ml})$ for $24 \mathrm{~h}$.

Collection of human samples. All experiments involving human subjects were approved by the Ethics Committee of the Chengdu Military General Hospital, and informed consent was provided by all subjects. Blood samples were obtained from 10 male patients with RA (age, 54.3 \pm 3.2 years) and from 10 healthy male donors (age, 52.5 \pm 3.6 years). Peripheral blood monocytes were isolated for the subsequent analysis of mRNA or protein expression. The plasma was subjected to enzyme-linked immunosorbent assay (ELISA) analysis to detect IL-1 $\beta$ expression. Patients enrolled to the present study were originally admitted to the Chengdu Military General Hospital (Chengdu, Sichuan, China) from January to June in 2015.

Gain or loss of function studies. Primary peritoneal macrophages were isolated and seeded into a 6-well plate at $80-90 \%$ density, and maintained at $37^{\circ} \mathrm{C}$ in a humidified $5 \% \mathrm{CO}_{2}$ atmosphere $10 \mathrm{~h}$ before transfection. Overexpression of miR-33 was achieved by transfecting the mouse peritoneal macrophages with a miR-33 mimic. Inhibition of miR-33 was achieved by transfecting the macrophages with an anti-miR-33. A scrambled miR was used as a control. NLRP3 expression in macrophages was silenced using a pre-designed small interfering (si)RNA sequence targeting NLRP3 (cat. no. 4390771; Thermo Fisher Scientific, Inc.). The negative siRNA control was also provided commercially (cat. 
no. 4390843; Thermo Fisher Scientific, Inc.). For macrophage transfection, the working concentration of the miRs was $100 \mathrm{nM}$, and for the siRNAs it was $20 \mathrm{nmol} / \mathrm{ml}$. The transfection reagent was Lipofectamine 2000 (cat. no. 11668500; Thermo Fisher Scientific, Inc.). The cells were incubated with miR-33, anti-miR-33, si-NLRP3 or the scramble for $5 \mathrm{~h}$, and then stimulated with PBS $(1,000 \mathrm{X})$ or LPS $(50 \mathrm{ng} / \mathrm{ml})$ for $24 \mathrm{~h}$ prior to further analysis.

Protein extraction and immunoblotting assays. Mouse peritoneal macrophages were lysed with radioimmunoprecipitation Lysis and Extraction Buffer (cat. no. 89900; Thermo Fisher Scientific, Inc.) supplemented with $1 \%$ protease cocktails 1 , 2 and 3, and $1 \mathrm{mM}$ phosphatase inhibitors (all Sigma-Aldrich; Merck KGaA, Darmstadt, Germany) and shaken for $30 \mathrm{~min}$ prior to centrifugation at $12,000 \mathrm{x}$ g for $30 \mathrm{~min}$ at $4^{\circ} \mathrm{C}$. The supernatant was then collected and quantified using a bicinchoninic acid assay kit (Roche Applied Science, Penzberg, Germany). The extracted proteins (50 $\mathrm{mg} /$ well) were separated via SDS-PAGE on a $10 \%$ gel, and transferred to a polyvinylidene difluoride membrane. The membrane was blocked with $5 \%$ non-fat milk at $4^{\circ} \mathrm{C}$ overnight, and then incubated with anti-NLRP3 (cat. no. 15101, dilution 1:1,000; Cell Signaling Technology, Inc., Danvers, MA, USA), anti-caspase-1 p20 (cat. no. 1780, dilution 1:1,000; Santa Cruz Biotechnology, Inc., Dallas, TX, USA) or anti- $\beta$-actin (cat. no. 8457, dilution 1:1,000; Cell Signaling Technology, Inc.) for $10 \mathrm{~h}$ at $4^{\circ} \mathrm{C}$. The membrane was rinsed 3 times with PBS containing $0.1 \%$ Tween-20, and incubated for $1 \mathrm{~h}$ with the appropriate horseradish peroxidase-conjugated secondary antibodies (cat. nos. A0181 and A0208, dilution 1:1,000; Beyotime Institute of Biotechnology) at $37^{\circ} \mathrm{C}$. The membrane was then washed with PBS containing 0.1\% Tween-20 and incubated with enhanced chemiluminescence substrate (cat. no. NEL105001EA; PerkinElmer, Inc., Waltham, MA, USA) for $1 \mathrm{~min}$ at $4^{\circ} \mathrm{C}$. The signals were captured using a ChemiDoc MP System (cat. no. 170-8280; Bio-Rad Laboratories, Inc., Hercules, CA, USA).

Reverse transcription-quantitative polymerase chain reaction (RT-qPCR). Total RNA from the mouse peritoneal macrophages and human blood monocytes was isolated using TRIzol reagent (Invitrogen; Thermo Fisher Scientific, Inc.). RNA ( $1 \mathrm{mg}$ ) was reverse transcribed into cDNA using the Omniscript RT kit (Qiagen GmbH, Hilden, Germany), according to the manufacturer's protocol.qPCR was performed using a 7900HT Fast Real-Time PCR system (Applied Biosystems; Thermo Fisher Scientific, Inc.). The mRNA expression levels were normalized to $\beta$-actin. Reactions were performed in duplicate using Applied Biosystems TaqMan Gene Expression assays and Universal PCR Master Mix (Applied Biosystems; Thermo Fisher Scientific, Inc.) The probes (human NLRP3, cat. no. Hs00366465_m1; human IL-1 $\beta$, cat. no. Hs01555410_m1; human $\beta$-actin, cat. no. Hs99999903_m1; mouse NLRP3, cat. no. Mm04210227_ g1; mouse IL-1 $\beta$, cat. no. Mm00434228_m1; mouse $\beta$-actin, cat. no. Mm99999915_g1) were provided commercially by Applied Biosystems; Thermo Fisher Scientific, Inc. The thermocycling conditions were as follows: Denaturation at $95^{\circ} \mathrm{C}$, followed by 40 cycles of denaturation at $95^{\circ} \mathrm{C}$ for $15 \mathrm{sec}$ and annealing at $60^{\circ} \mathrm{C}$ for $1 \mathrm{~min}$. Relative target gene expression was calculated using the $2^{-\Delta \Delta \mathrm{Cq}}$ method (23).

ROS assays. miR-33 mimic or anti-miR-33-transfected peritoneal macrophages were seeded in 96-well plates (80-90\% fusion) and treated with $50 \mu \mathrm{M}$ 5-(and-6)-chloromethyl-2',7'-dichlorodihydrofluorescein diacetate acetyl ester (DCFDA; cat. no. D6883; Sigma-Aldrich; Merck KGaA) for $6 \mathrm{~h}$ at $37^{\circ} \mathrm{C}$. ROS production was determined by measuring the level of DCFDA hydrolysis to fluorescent 2',7'-dichlorofluorescein, which is promoted by a number of reactive radical species, and allows for the assessment of general oxidative stress. DCFDA conversion was kinetically measured at 0, 2, 4 and 6 h using a microplate reader (BioTek Instruments, Inc., Winooski, VT, USA) at $488 \mathrm{~nm}$ excitation and $535 \mathrm{~nm}$ emission wavelengths.

Caspase-1 activity assay. Caspase-1 activity was measured using the Caspase-1/ICE Colorimetric Assay kit (cat. no. BF15100; R\&D Systems, Inc., Minneapolis, MN, USA), according to the manufacturer's instructions. The macrophages and human blood monocytes were lysed in the kit lysis buffer and diluted to a final protein concentration of $2-4 \mathrm{mg} / \mathrm{ml}$. The enzymatic reaction was performed in a 96-well flat-bottom microplate. Each reaction contained $50 \mu 1$ cell lysate and $50 \mu 12 \mathrm{X}$ reaction buffer, to which $5 \mu \mathrm{l}$ caspase- 1 colorimetric substrate was added and incubated at $37^{\circ} \mathrm{C}$ for $1.5 \mathrm{~h}$. The plate was read using a microplate reader at a wavelength of $405 \mathrm{~nm}$. The activity of caspase-1 was displayed as optical density (OD)405/mg protein.

Mitochondrial biogenesis and function assays. Mitochondrial oxygen consumption rates (OCRs) of the scramble miR or miR-33 mimic-transfected macrophages were measured following different treatments in 96-well plates $(10,000$ cells/well) using a Seahorse XF Cell Mito Stress Test kit (cat. no. 101706-100; Seahorse Bioscience; Agilent Technologies, Inc.) on the XFe96 Extracellular Flux Analyzer (Seahorse Bioscience; Agilent Technologies, Inc.) according to the manufacturer's instructions. Briefly, basal cellular OCRs were recorded in the absence of metabolic inhibitors or uncouplers. ATP synthase was inhibited with $2 \mu \mathrm{g} / \mathrm{ml}$ oligomycin, followed by uncoupling of the respiratory chain from oxidative phosphorylation by stepwise titration with $1 \mu \mathrm{g} / \mathrm{ml}$ FCCP to achieve maximal OCRs. Subsequently, rotenone (Mito Inhibitor B), a complex I inhibitor $(1 \mu \mathrm{g} / \mathrm{ml})$, and antimycin A (Mito Inhibitor A), a complex III inhibitor (1 $\mu \mathrm{g} / \mathrm{ml}$ ), were combined to completely inhibit the mitochondrial respiratory chain. The results are presented as the OCR (pmol/min).

ELISA analysis. IL-1 $\beta$ levels in the supernatants of cultured macrophages were measured using a IL-1 $\beta$ ELISA kit (cat. no. MLB00C; R\&D Systems, Inc.), and IL-1 $\beta$ levels in blood plasma were measured using another ELISA kit (cat. no. DLB50; R\&D Systems, Inc.), according to the manufacturer's instructions. The final cytokine concentration in the supernatants of cultured cells was normalized to the total number of cells. The mouse or human IL-1 $\beta$ standard was included in the corresponding kit. These tests were performed 
A

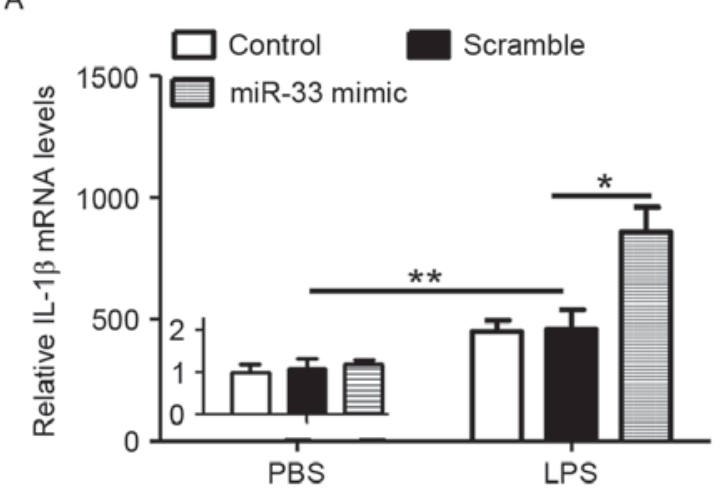

B

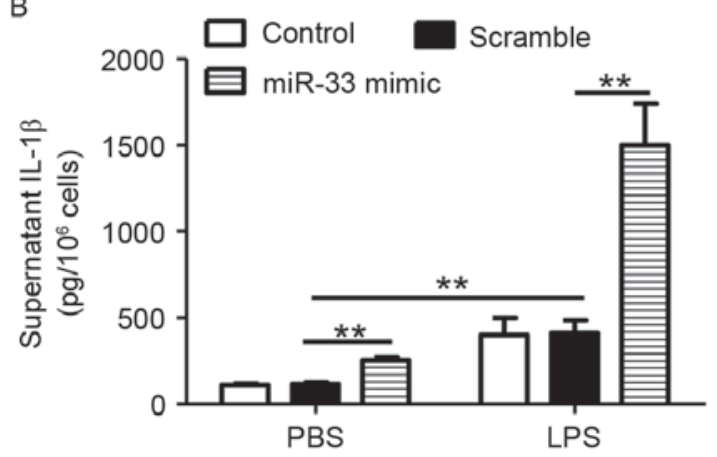

C

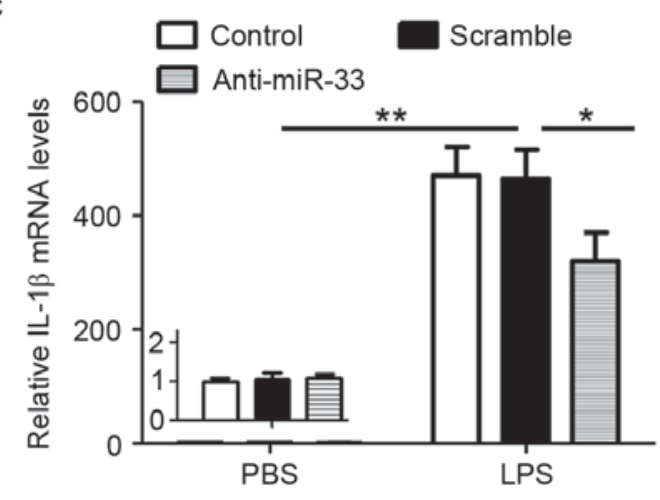

D

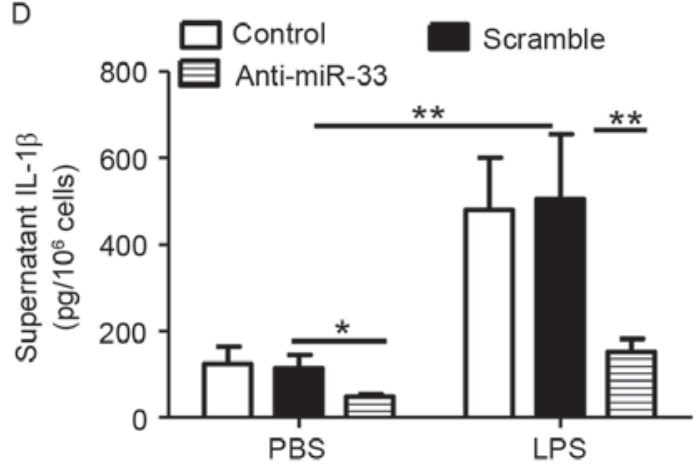

Figure 1. miR-33 increases the secretion of IL-1 $\beta$ in primary macrophages. (A) The relative mRNA and (B) supernatant IL-1 $\beta$ levels in untreated control mouse peritoneal macrophages or those that were transfected with a scramble miR (100 $\mathrm{nM})$ or a mimic of miR-33 (100 nM) for $5 \mathrm{~h}$, followed by treatment with LPS (50 ng/ml) or PBS as a control for $24 \mathrm{~h}$. (C) The relative mRNA and (D) supernatant IL-1 $\beta$ levels in untreated control mouse peritoneal macrophages or those transfected with a scramble miR $(100 \mathrm{nM})$ or an anti-miR-33 (100 nM) for $5 \mathrm{~h}$, followed by treatment with LPS (50 ng/ml) or PBS as a control for $24 \mathrm{~h}$. The results are presented as the mean \pm standard error of the mean $(\mathrm{n}=4)$. ${ }^{*} \mathrm{P}<0.05$ and ${ }^{* *} \mathrm{P}<0.01$, as indicated. miR, microRNA; IL-1 $\beta$, interleukin-1 $\beta$; LPS, lipopolysaccharide; PBS, phosphate-buffered saline.

using a microplate reader (BioTek Instruments, Inc.) set to $450 \mathrm{~nm}$.

Statistical analysis. Statistical analysis was performed using GraphPad Prism version 5.01 (GraphPad Software, Inc., La Jolla, CA, USA). The results are expressed as the mean \pm standard error of the mean and were analyzed using one-way analysis of variance followed by the Tukey post hoc test for multiple comparisons, and a two-tailed unpaired Student's t-test. $\mathrm{P}<0.05$ was considered to indicate a statistically significant difference.

\section{Results}

miR-33 potentiates the secretion of IL-1 $\beta$ in primary macrophages. In order to determine whether miR-33 regulates the inflammasome pathway, the expression of IL-1 $\beta$ in miR-33 deficient or proficient macrophages was measured. To increase inflammation signaling, LPS was also employed. As presented in Fig. 1A and B, treatment with a miR-33 mimic significantly increased LPS-stimulated IL- $1 \beta$ expression at the mRNA and protein levels when compared with scramble miR-transfected controls. By contrast, miR-33 did not stimulate IL-1 $\beta$ mRNA levels in the absence of LPS treatment (Fig. 1A), however, it significantly induced IL-1 $\beta$ secretion (Fig. 1B). Consistent with these observations, treatment of macrophages with anti-miR-33 and/or LPS resulted in the inverse effects on IL- $1 \beta$ expression and secretion (Fig. 1C and D). Therefore, the results indicated that miR-33 may increase IL-1 $\beta$ secretion by stimulating the inflammasome pathway.

miR-33 increases the expression of NLRP3. As shown in Fig. 2A, transfection of macrophages with the miR-33 mimic significantly stimulated NLRP3 mRNA expression in cells with or without LPS treatment when compared with scramble miR-transfected controls. Consistently, anti-miR-33 significantly attenuated the expression levels of NLRP3 mRNA in PBS or LPS-treated macrophages when compared with scramble miR-transfected controls (Fig. 2B). In addition, the results confirmed that treatment with a miR-33 mimic or anti-miR-33 induced or attenuated NLRP3 protein expression in peritoneal macrophages, respectively (Fig. 2C and D).

miR-33 increases the activity of caspase-1. The NLRP3 inflammasome activates caspase- 1 , and caspase- 1 cleaves pro-IL-1 $\beta$ protein to produce mature IL- $1 \beta$, which is subsequently secreted $(2,5)$. Therefore, the present study investigated the role of miR-33 in regulating caspase-1 activity. Treatment of PBS or LPS-stimulated peritoneal macrophages with the miR-33 mimic significantly induced the activity of caspase-1 when compared with scramble miR-transfected controls (Fig. 3A). Consistent with this result, the expression of the active form of caspase-1, p20, was increased by miR-33 
A

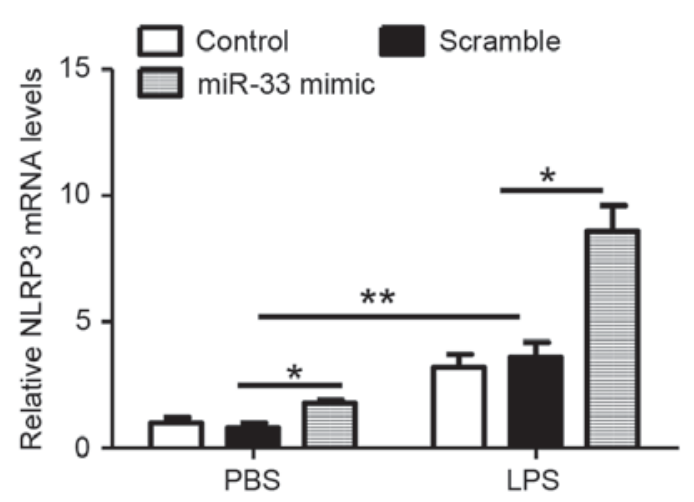

C

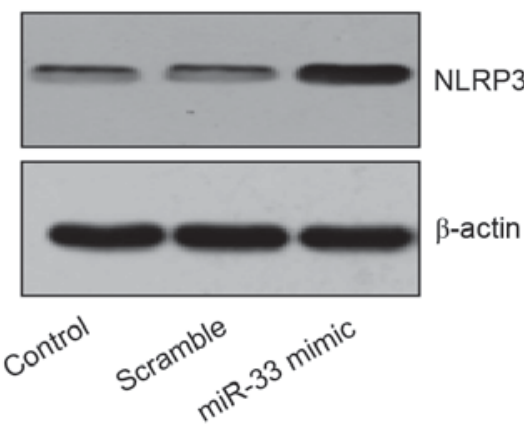

B

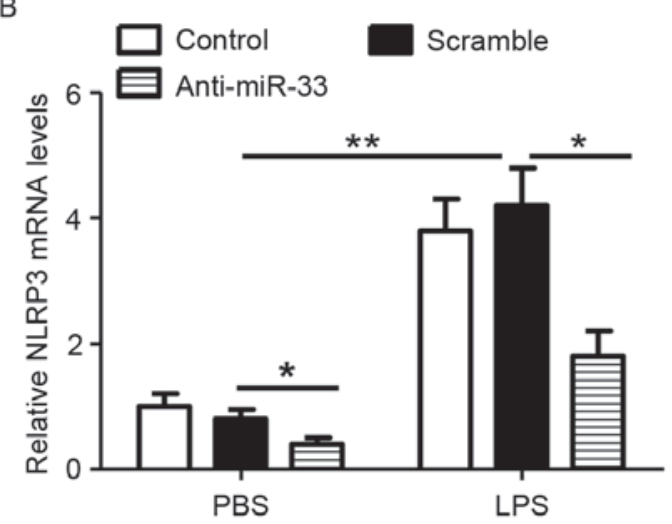

D
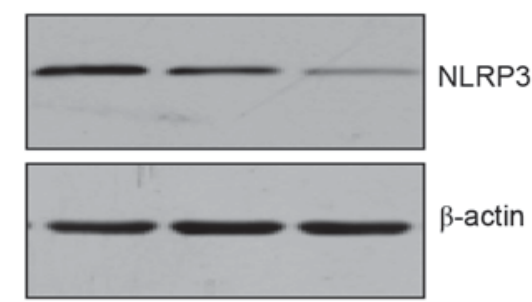

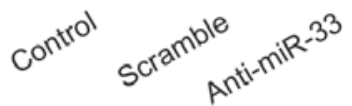

Figure 2. miR-33 increases the expression of NLRP3. (A) The relative mRNA levels of NLRP3 in untreated control mouse peritoneal macrophages or those transfected with a scramble miR (100 nM) or an miR-33 mimic (100 nM). (B) The relative mRNA levels of NLRP3 in untreated control mouse peritoneal macrophages or those transfected with a scramble miR $(100 \mathrm{nM})$ or anti-miR-33 (100 nM). At $5 \mathrm{~h}$ following transfection with miR-33 mimics, anti-miR-33 or scramble controls, cells were treated with LPS (50 ng/ml) or PBS. (C) Immunoblotting assay of NLRP3 in untreated control mouse peritoneal macrophages or those or transfected with a scrambled $\mathrm{miR}(100 \mathrm{nM})$ or an miR-33 mimic (100 nM). (D) Immunoblotting assay of NLRP3 in untreated control mouse peritoneal macrophages or those transfected with a scramble miR $(100 \mathrm{nM})$ or anti-miR-33 (100 nM). At $5 \mathrm{~h}$ following transfection with miR-33 mimics, anti-miR-33 or scramble miR, cells were treated with LPS $(50 \mathrm{ng} / \mathrm{ml})$ for $24 \mathrm{~h}$. The results are presented as the mean \pm standard error of the mean $(\mathrm{n}=4)$. ${ }^{*} \mathrm{P}<0.05$ and ${ }^{* *} \mathrm{P}<0.01$, as indicated. NLRP3, nucleotide binding domain and leucine-rich repeat pyrin 3 domain; miR, microRNA; LPS, lipopolysaccharide; PBS, phosphate-buffered saline.

transfection when compared with the controls (Fig. 3B). By contrast, transfection with anti-miR-33 attenuated caspase-1 activity in PBS and LPS-stimulated cells (Fig. 3C). These results indicated that $\mathrm{miR}-33$ may regulate the inflammasome pathway in macrophages. As demonstrated in Fig. 3D, transfection of macrophages with si-NLRP3 effectively silenced NLRP3 protein expression. In addition, miR-33 was observed to induce caspase- 1 activity and IL- $1 \beta$ secretion in an NLRP3-dependent manner (Fig. 3E and F).

miR-33 attenuates mitochondrial oxygen consumption and increases the production of cellular ROS. According to previous studies, ROS stimulate NLRP3 expression $(3,5)$. Therefore, the present study investigated whether miR-33 regulates ROS production in macrophages. As presented in Fig. 4A and B, treatment with an miR-33 mimic significantly induced cellular ROS production in peritoneal macrophages with or without LPS stimulation when compared with scramble miR controls. Consistent with these observations, treatment with anti-miR-33 significantly decreased ROS production when compared with controls (Fig. 4C and D). Mitochondrial function was analyzed in the present study, as mitochondria are known to be a major source of ROS. It was demonstrated that treatment with the miR-33 mimic significantly decreased the basal OCR in mitochondria when compared with scramble miR-transfected controls (Fig. 4E). ROS in macrophages were significantly increased in response to treatment with the miR-33 mimic at basal levels (Fig. 4F). Treatment with the NAC or GSH antioxidants efficiently reduced cellular ROS (Fig. 4F). The results of the present study indicate that miR-33-induced mitochondrial dysfunction may account for the increased production of cellular ROS in macrophages.

miR-33 stimulates the inflammasome pathway by impairing mitochondrial function and inducing ROS production in peritoneal macrophages. In order to further verify the role of miR-33-mediated mitochondrial function and ROS production in inflammasome activation, NLRP3 expression, caspase-1 activity and IL-1 $\beta$ secretion were analyzed in peritoneal macrophages treated with a miR-33 mimic, antioxidants (NAC and GSH) or the mitochondrial stimulator FCCP. The results demonstrated that miR-33-induced NLRP3 expression was prevented by NAC treatment (Fig. 5A). In addition, transfection with the miR-33 mimic induced caspase-1 activity, whereas additional treatment with antioxidants or FCCP abolished this effect (Fig. 5B). Furthermore, miR-33 mimic-induced IL-1 $\beta$ secretion was prevented by treatment with NAC, GSH or FCCP (Fig. 5C). 
A

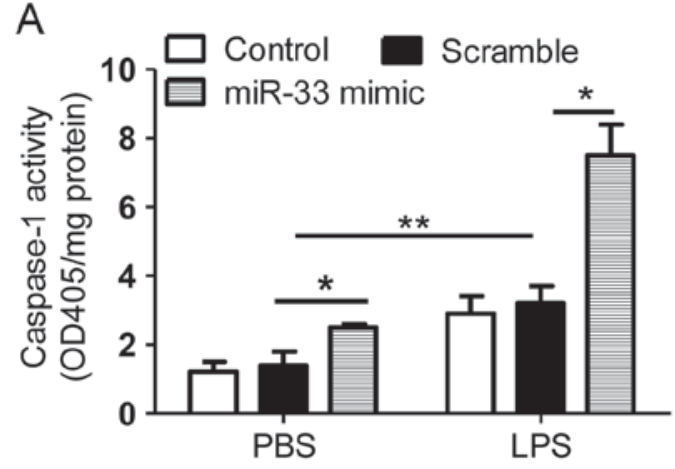

C
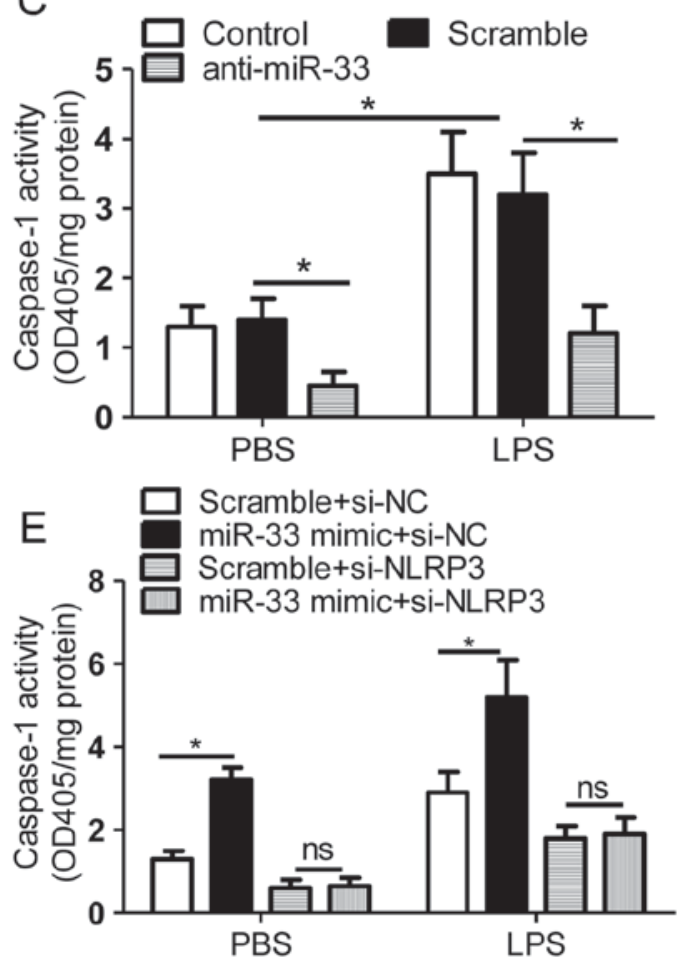

$\mathrm{B}$
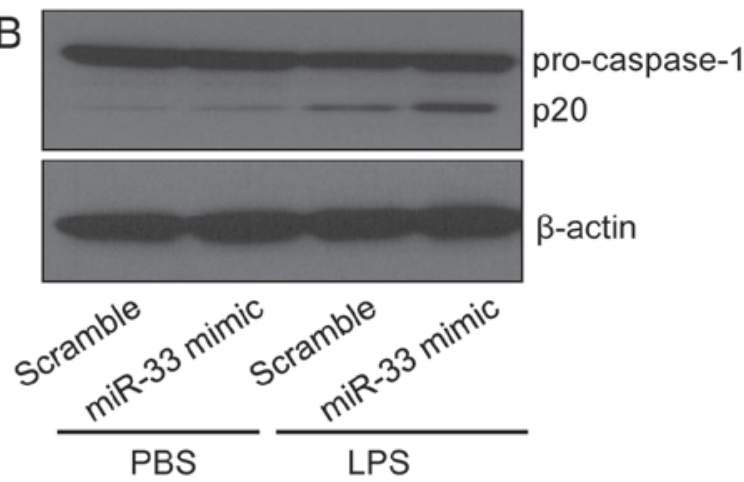

D
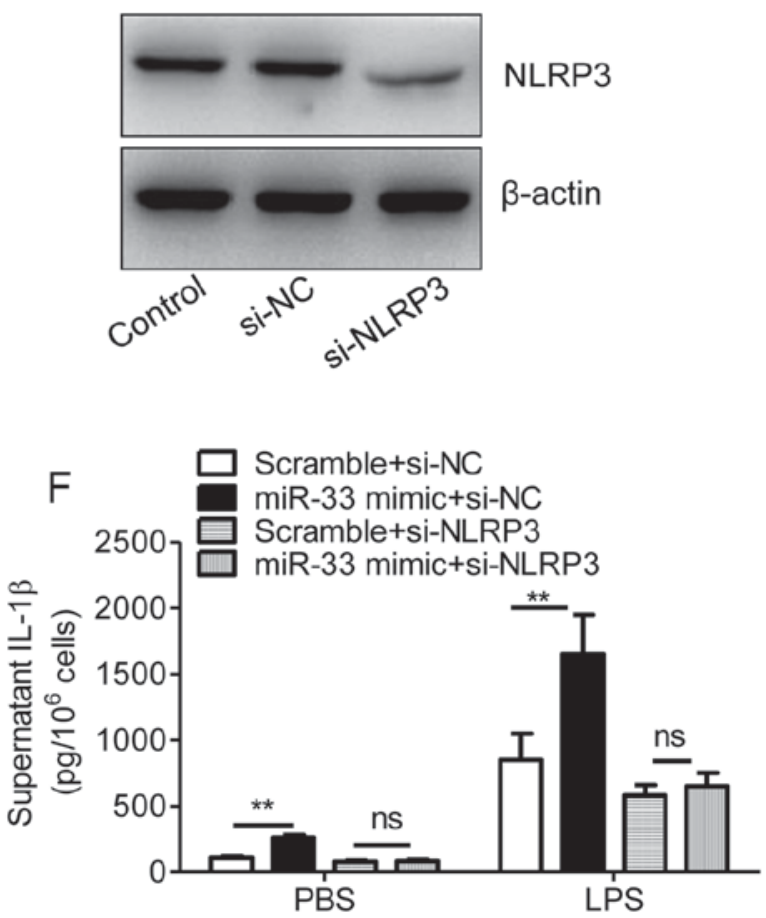

Figure 3. miR-33 increases the activity of caspase-1. (A) The activity of caspase-1 in untransfected control mouse peritoneal macrophages or those transfected with a scramble miR $(100 \mathrm{nM})$ or an miR-33 mimic $(100 \mathrm{nM})$. (B) Immunoblotting assays for pro-caspase-1 expression in mouse peritoneal macrophages transfected with a scrambled miR $(100 \mathrm{nM})$ or an miR-33 mimic $(100 \mathrm{nM})$. (C) Activity of caspase-1 in untransfected control mouse peritoneal macrophages or those transfected with a scramble miR $(100 \mathrm{nM})$ or an anti-miR-33 $(100 \mathrm{nM})$. At 5 h following transfection with miR-33 mimics, anti-miR-33 or scramble controls, cells were treated with LPS $(50 \mathrm{ng} / \mathrm{ml})$ or PBS. (D) Immunoblotting assays for NLRP3 expression in mouse peritoneal macrophages transfected with si-NC $(20 \mathrm{nmol} / \mathrm{ml})$ or si-NLRP3 $(20 \mathrm{nmol} / \mathrm{ml})$ for $24 \mathrm{~h}$. (E) The activity of caspase-1 and (F) the supernatant IL-1 1 levels in mouse peritoneal macrophages transfected with a scrambled miR $(100 \mathrm{nM})$ or an miR-33 mimic $(100 \mathrm{nM})$ in addition to si-NC $(20 \mathrm{nmol} / \mathrm{ml})$ or si-NLRP3 $(20 \mathrm{nmol} / \mathrm{ml})$ for $5 \mathrm{~h}$, followed by treatment with LPS $(50 \mathrm{ng} / \mathrm{ml})$ or PBS for $24 \mathrm{~h}$. The results are presented as the mean \pm standard error of the mean $(\mathrm{n}=4)$. ${ }^{*} \mathrm{P}<0.05$ and ${ }^{* *} \mathrm{P}<0.01$, as indicated. miR, microRNA; LPS, lipopolysaccharide; PBS, phosphate-buffered saline; NLRP3, nucleotide binding domain and leucine-rich repeat pyrin 3 domain; siRNA, short interfering RNA; si-NC, negative control siRNA; IL-1 $\beta$, interleukin-1 $\beta$; ns, not significant.

Elevated miR-33 levels are associated with activation of the NLRP3 inflammasome pathway in RA-associated monocytes. The expression of miR-33 and activation of the NLRP3 inflammasome signaling pathway was analyzed in peripheral blood monocytes derived from patients with RA and compared with those from healthy donors, as IL-1 $\beta$ is a well-documented inducer of RA $(24,25)$. It was demonstrated that RA-associated monocytes expressed significantly increased miR-33 (Fig. 6A), NLRP3 (Fig. 6B) and IL-1 $\beta$ (Fig. 6C) levels when compared with monocytes from healthy donors. In addition, the activity of caspase-1 and the levels of plasma IL-1 $\beta$ were significantly increased in the RA-associated monocytes compared with control monocytes
(Fig. 6D and E). The results of the present study indicated a potential role for the miR-33/NLRP3 inflammasome/IL-1 $\beta$ axis in the development of RA.

\section{Discussion}

To the best of the author's knowledge, the present study is the first to suggest that miR-33 may function as a positive regulator of the NLRP3 inflammasome by repressing mitochondrial OCRs and inducing ROS accumulation in macrophages. In addition, the results demonstrated that the expression of miR-33 and the activity of the NLRP3 inflammasome were significantly increased in the blood monocytes of patients with 
A

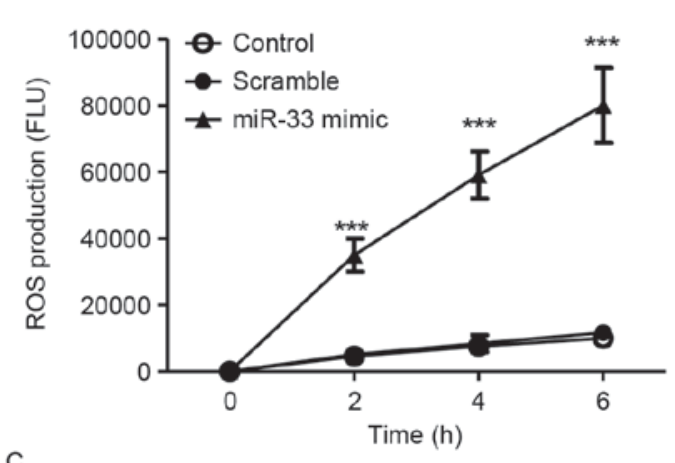

c
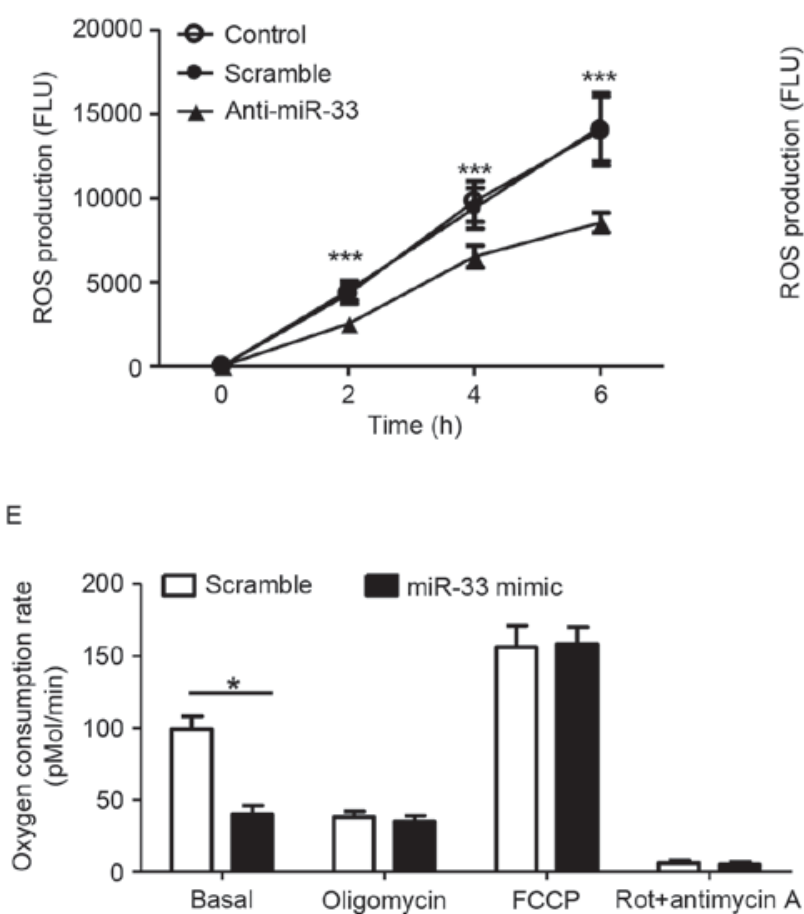

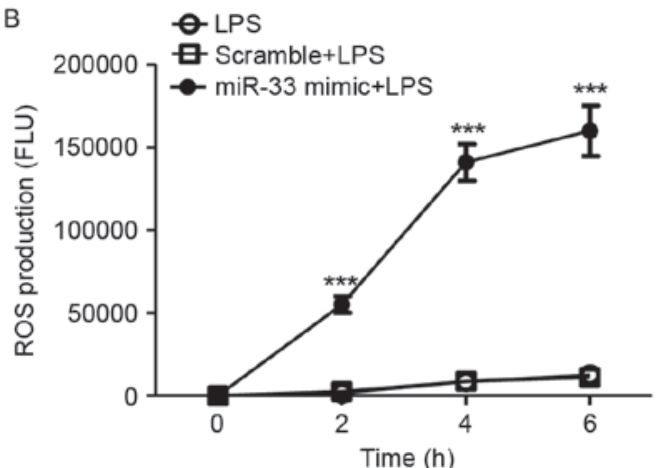

D
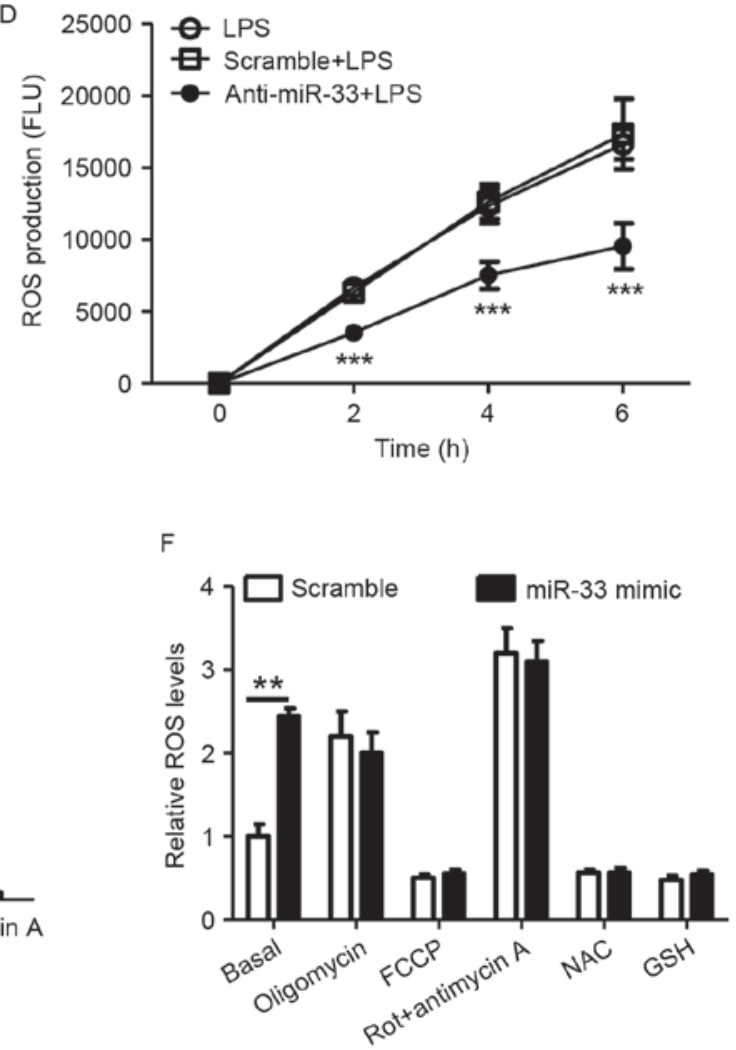

Figure 4. miR-33 attenuates mitochondrial oxygen consumption and increases the production of cellular ROS. (A) Cellular ROS production of peritoneal macrophages treated with a scramble miR $(100 \mathrm{nM})$ or an miR-33 mimic $(100 \mathrm{nM}) .^{* * *} \mathrm{P}<0.005$ vs. scramble. (B) Cellular ROS production of peritoneal macrophages that were treated with a scramble miR $(100 \mathrm{nM})$ or an miR-33 mimic (100 nM) for $5 \mathrm{~h}$, followed by treatment with LPS (50 ng/ml) for $24 \mathrm{~h}$. ${ }^{* * * *} \mathrm{P}<0.005$ vs. scramble + LPS. (C) Cellular ROS production of peritoneal macrophages treated with a scramble miR (100 $\left.\mathrm{nM}\right)$ or anti-miR-33 (100 nM). ${ }^{* * *} \mathrm{P}<0.005$, anti-miR-33 vs. scramble. (D) Cellular ROS production of peritoneal macrophages that were treated with a scramble miR (100 nM) or anti-miR-33 $(100 \mathrm{nM})$ for $5 \mathrm{~h}$, followed by treatment with LPS $(50 \mathrm{ng} / \mathrm{ml})$ for $24 \mathrm{~h}$. ${ }^{* * *} \mathrm{P}<0.005 \mathrm{vs}$. scramble + LPS. (E) The oxygen consumption rate of peritoneal macrophages treated with a scrambled miR $(100 \mathrm{nM})$ or an miR-33 mimic (100 $\mathrm{nM})$ for $24 \mathrm{~h}$, followed by mitochondrial function assays. (F) Relative ROS production in peritoneal macrophages that were transfected with a scramble miR (100 nM) or an miR-33 mimic (100 nM) and subsequently treated with various regulators of mitochondrial function or antioxidants for $24 \mathrm{~h}$. The results are presented as the mean \pm standard error of the mean $(\mathrm{n}=5)$. $\mathrm{P}<0.05$ and ${ }^{* *} \mathrm{P}<0.01$, as indicated. miR, microRNA; ROS, reactive oxygen species; LPS, lipopolysaccharide; Rot, rotenone; FCCP, carbonyl cyanide p-(trifluoromethoxy) phenylhydrazone; NAC, $N$-acetylcysteine; GSH, glutathione.

RA when compared with healthy donors. The results of the present study revealed a potential role for miR-33 in chronic inflammatory diseases, such as RA.

In the present study, miR-33 was observed to repress the mitochondrial OCR and increase ROS production. A previous study demonstrated that anti-miR-33 de-repressed peroxisome proliferator-activated receptor $\gamma$ coactivator $1-\alpha$ (PGC1- $\alpha$ ), pyruvate dehydrogenase kinase 4 and solute carrier family 25 member 25 target genes, and enhanced mitochondrial respiration (21). It was hypothesized that miR-33 inhibited the OCR rate by repressing PGC1- $\alpha$ expression, as PGC $1-\alpha$ is a well-documented stimulator of mitochondrial biogenesis (26). Therefore, mitochondrial biogenesis-associated genes require further analysis in future studies to verify this hypothesis. The results of the present study demonstrated that ROS accumulation was due to decreased oxygen consumption in the mitochondria, which was consistent with results from previous studies $(5,27)$.

miR-33 may regulate proinflammatory cytokine production via a number of different signaling pathways. For instance, cholesterol is an important inducer of inflammatory responses (28), and miR-33 regulates cholesterol 
A

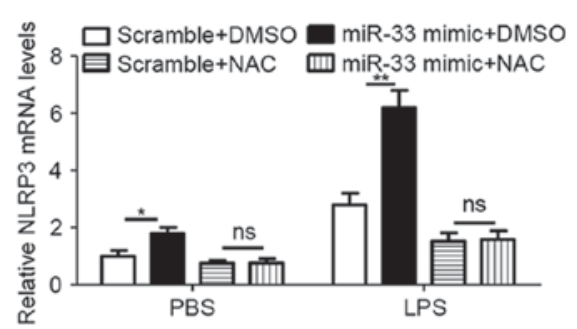

$\mathrm{C}$
B

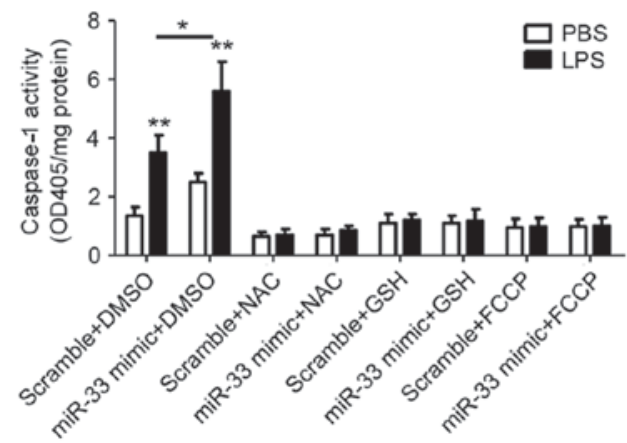

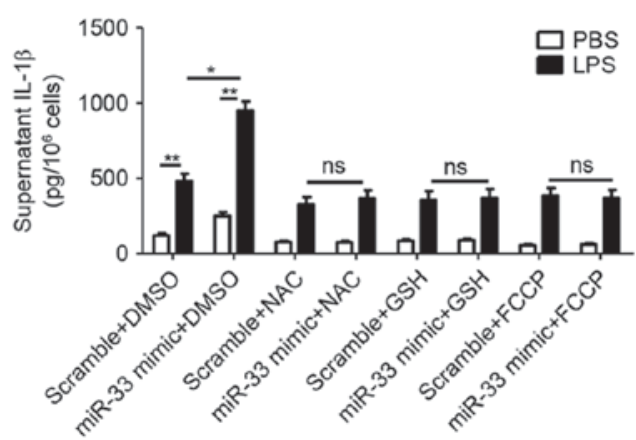

Figure 5. miR-33 stimulates the inflammasome signaling pathway by impairing mitochondrial function and inducing ROS production in peritoneal macrophages. (A) The mRNA levels of NLRP3 in mouse peritoneal macrophages that were transfected with a scramble miR (100 $\mathrm{nM})$ or an miR-33 mimic (100 nM) for $5 \mathrm{~h}$, followed by treatment with LPS $(50 \mathrm{ng} / \mathrm{ml})$ or PBS as a control, plus NAC (20 mM) or DMSO for $24 \mathrm{~h}$. (B) The activity of caspase-1 and (C) the level of IL-1 $\beta$ in the supernatant of mouse peritoneal macrophages that were transfected with a scramble miR $(100 \mathrm{nM})$ or miR-33 mimic $(100 \mathrm{nM})$ for $5 \mathrm{~h}$, followed by treatment with LPS $(50 \mathrm{ng} / \mathrm{ml})$ or PBS as a control, plus NAC $(20 \mathrm{mM})$, GSH $(10 \mathrm{mM})$ or FCCP $(1 \mu \mathrm{g} / \mathrm{ml})$ for $24 \mathrm{~h}$. The results are presented as the mean \pm standard error of the mean $(n=4) .{ }^{*} \mathrm{P}<0.05$ and ${ }^{* *} \mathrm{P}<0.01$, as indicated. miR, microRNA; NLRP3, nucleotide binding domain and leucine-rich repeat pyrin 3 domain; LPS, lipopolysaccharide; PBS, phosphate-buffered saline; NAC, $N$-acetylcysteine; DMSO, dimethyl sulfoxide; IL-1 $\beta$, interleukin-1 $\beta$; GSH, glutathione; FCCP, carbonyl cyanide p-(trifluoromethoxy)phenylhydrazone; OD, optical density; ns, not significant.

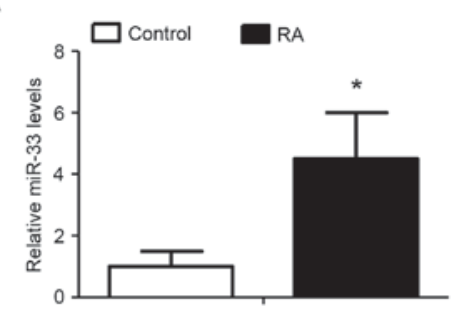

C

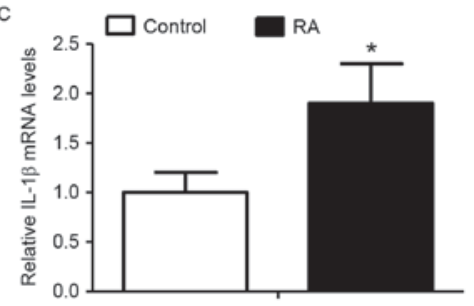

$\mathrm{E}$

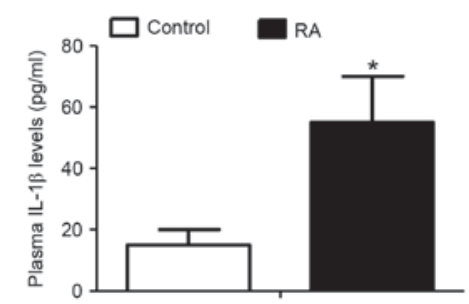

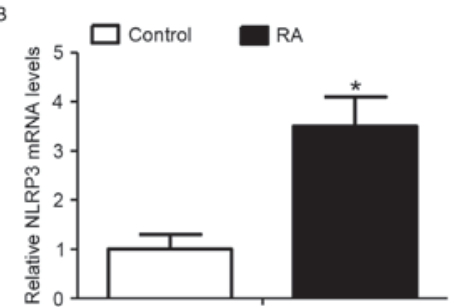

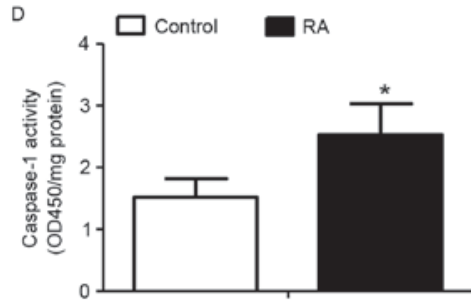

Figure 6. Elevated miR-33 levels are associated with activation of the NLRP3 inflammasome pathway in RA-associated monocytes. Relative (A) miR-33, (B) NLRP3 and (C) IL-1 $\beta$ mRNA levels in peripheral blood monocytes from patients with RA compared with controls. (D) Activity of caspase-1 in monocytes from patients with RA compared with controls. (E) Plasma IL-1 $\beta$ levels in patients with RA compared with controls. The results are presented as the mean \pm standard error of the mean $(n=10)$. ${ }^{*} \mathrm{P}<0.05 \mathrm{vs}$. control. RA, rheumatoid arthritis; miR, microRNA; NLRP3, nucleotide binding domain and leucine-rich repeat pyrin 3 domain; IL-1 $\beta$, interleukin-1 $\beta$; controls, healthy donors; OD, optical density. 
efflux in macrophages (19). In addition, the 5'-adenosine monophosphate-activated protein kinase signaling pathway, which is regulated by miR-33 (22), regulates macrophage polarization (29). Ho et al (30) reported that miR-33 repressed receptor-interacting protein 140-dependent proinflammatory cytokine production, which contradicts the results of the present study. There are a number of differences in the treatment conditions between this previous study and the present study, including the macrophage cell type (Raw264.7 cells vs. primary macrophages) and LPS treatment conditions (4 vs. $24 \mathrm{~h} ; 20 \mathrm{ng} / \mathrm{ml}$ vs. $50 \mathrm{ng} / \mathrm{ml}$ ). It is possible that miR-33 may regulate toll-like receptor 4 (TLR-4) signaling, thereby further potentiating NLRP3 expression and IL-1 $\beta$ production. However, in the present study it was observed that miR-33 induced IL-1 $\beta$ protein levels independently of TLR-4 expression (data not shown). The results presented indicated that miR-33 may regulate a complex signaling network. However, the respective contribution of each pathway downstream of miR-33 in proinflammatory cytokine production remains to be elucidated. The results of the present study reflected a long-term effect of miR-33, based on its roles in regulating mitochondrial function and ROS production. The miR-33-regulated cholesterol signaling pathway may be important for the development of atherosclerosis, whereas the NLRP3 inflammasome pathway may be associated with the pathogenesis of RA.

In the present study, it was demonstrated that the NLRP3 inflammasome was activated in RA-associated monocytes in human subjects, which is consistent with the results from a recent clinical study (31). In addition, the present study demonstrated that miR-33 levels were significantly increased in the blood monocytes of patients with RA when compared with healthy control subjects, suggesting that the miR-33/NLRP3 inflammasome axis may regulate RA progression and that miR-33 may be a novel marker for the disease. In order to further verify the results of the present study, future animal model and clinical studies are required.

In conclusion, miR-33 was identified to be a positive regulator of the NLRP3 inflammasome in macrophages, and the miR-33/NLRP3 inflammasome signaling pathway may be associated with the development of RA.

\section{Acknowledgements}

The present study was supported in part by the National Nature Science Foundation of China (grant no. 81001336), the Sichuan Provincial Health Department Foundation (grant nos. 130320 and 130322) and the Chengdu Military General Hospital Foundation (grant no. 2013YG-B037/B096). NPG Language Editing provided an English language service for the present study.

\section{References}

1. Strowig T,Henao-Mejia J, Elinav E and Flavell R: Inflammasomes in health and disease. Nature 481: 278-286, 2012.

2. Eisenbarth SC, Colegio OR, O'Connor W, Sutterwala FS and Flavell RA: Crucial role for the Nalp3 inflammasome in the immunostimulatory properties of aluminium adjuvants. Nature 453: 1122-1126, 2008

3. Schroder K, Zhou R and Tschopp J: The NLRP3 inflammasome: A sensor for metabolic danger? Science 327: 296-300, 2010.
4. Zhou R, Yazdi AS, Menu P and Tschopp J: A role for mitochondria in NLRP3 inflammasome activation. Nature 469: 221-225, 2011.

5. Miao H, Ou J, Ma Y, Guo F, Yang Z, Wiggins M, Liu C, Song W, Han X, Wang M, et al: Macrophage CGI-58 deficiency activates ROS-inflammasome pathway to promote insulin resistance in mice. Cell Rep 7: 223-235, 2014.

6. Siebert S, Tsoukas A, Robertson J and McInnes I: Cytokines as therapeutic targets in rheumatoid arthritis and other inflammatory diseases. Pharmacol Rev 67: 280-309, 2015.

7. Cunnane G, Madigan A, Murphy E, FitzGerald O and Bresnihan B: The effects of treatment with interleukin-1 receptor antagonist on the inflamed synovial membrane in rheumatoid arthritis. Rheumatology (Oxford) 40: 62-69, 2001.

8. Ma Z, Wang B, Wang M, Sun X, Tang Y, Li M, Li F and Li X: TL1A increased IL- 6 production on fibroblast-like synoviocytes by preferentially activating TNF receptor 2 in rheumatoid arthritis. Cytokine 83: 92-98, 2016

9. Fontalba A, Martinez-Taboada V, Gutierrez O, Pipaon C, Benito N, Balsa A, Blanco R and Fernandez-Luna JL: Deficiency of the NF-kappaB inhibitor caspase activating and recruitment domain 8 in patients with rheumatoid arthritis is associated with disease severity. J Immunol 179: 4867-4873, 2007.

10. Kastbom A, Verma D, Eriksson P, Skogh T, Wingren G and Söderkvist P: Genetic variation in proteins of the cryopyrin inflammasome influences susceptibility and severity of rheumatoid arthritis (the Swedish TIRA project). Rheumatology (Oxford) 47: 415-417, 2008.

11. Ben Hamad M, Cornelis F, Marzouk S, Chabchoub G, Bahloul Z, Rebai A, Fakhfakh F, Ayadi H, Petit-Teixeira E and Maalej A: Association study of CARD8 (p.C10X) and NLRP3 (p.Q705K) variants with rheumatoid arthritis in French and Tunisian populations. Int J Immunogenet 39: 131-136, 2012.

12. Walle LV, Van Opdenbosch N, Jacques P, Fossoul A, Verheugen E, Vogel P, Beyaert R, Elewaut D, Kanneganti TD, van Loo G and Lamkanfi M: Negative regulation of the NLRP3 inflammasome by A20 protects against arthritis. Nature 512: 69-73, 2014.

13. Wen H, Gris D, Lei Y, Jha S, Zhang L, Huang MT, Brickey WJ and Ting JP: Fatty acid-induced NLRP3-ASC inflammasome activation interferes with insulin signaling. Nat Immunol 12: 408-415, 2011.

14. Heneka MT, Kummer MP, Stutz A, Delekate A, Schwartz S, Vieira-Saecker A, Griep A, Axt D, Remus A, Tzeng TC, et al: NLRP3 is activated in Alzheimer's disease and contributes to pathology in APP/PS1 mice. Nature 493: 674-678, 2013.

15. Wree A, Eguchi A, McGeough MD, Pena CA, Johnson CD, Canbay A, Hoffman HM and Feldstein AE: NLRP3 inflammasome activation results in hepatocyte pyroptosis, liver inflammation, and fibrosis in mice. Hepatology 59: 898-910, 2014.

16. Shalgi R, Lieber D, Oren M and Pilpel Y: Global and local architecture of the mammalian microRNA-transcription factor regulatory network. PLoS Comput Biol 3: e131, 2007.

17. Ryazansky SS, Gvozdev VA and Berezikov E: Evidence for post-transcriptional regulation of clustered microRNAs in Drosophila. BMC Genomics 12: 371, 2011.

18. Krützfeldt J and Stoffel M: MicroRNAs: A new class of regulatory genes affecting metabolism. Cell Metab 4: 9-12, 2006.

19. Rayner KJ, Suárez Y, Dávalos A, Parathath S, Fitzgerald ML, Tamehiro N, Fisher EA, Moore KJ and Fernández-Hernando C: MiR-33 contributes to the regulation of cholesterol homeostasis. Science 328: 1570-1573, 2010.

20. Esau CC, Hussain FN, McDaniel AL, Marshall SM, van Gils JM, Ray TD, Sheedy FJ, Goedeke L, Liu X, Khatsenko OG, et al: Inhibition of miR-33a/b in non-human primates raises plasma HDL and lowers VLDL triglycerides. Nature 478: 404-407, 2011.

21. Karunakaran D, Thrush AB, Nguyen MA, Richards L, Geoffrion M, Singaravelu R, Ramphos E, Shangari P, Ouimet M, Pezacki JP, et al: Macrophage mitochondrial energy status regulates cholesterol efflux and is enhanced by Anti-miR33 in atherosclerosis. Circ Res 117: 266-278, 2015.

22. Ouimet M, Ediriweera HN, Gundra UM, Sheedy FJ, Ramkhelawon B, Hutchison SB, Rinehold K, van Solingen C, Fullerton MD, Cecchini K, et al: MicroRNA-33-dependent regulation of macrophage metabolism directs immune cell polarization in atherosclerosis. J Clin Invest 125: 4334-4348, 2015.

23. Livak KJ and Schmittgen TD: Analysis of relative gene expression data using real-time quantitative PCR and the 2(-Delta Delta C(T)) Method. Methods 25: 402-408, 2001. 
24. Eklund KK, Leirisalo-Repo M, Ranta P, Mäki T, Kautiainen H, Hannonen P, Korpela M, Hakala M, Järvinen P and Möttönen T: Serum IL-1beta levels are associated with the presence of erosions in recent onset rheumatoid arthritis. Clin Exp Rheumatol 25: 684-689, 2007.

25. Ruscitti P, Cipriani P, Carubbi F, Liakouli V, Zazzeroni F, Di Benedetto P, Berardicurti O, Alesse E and Giacomelli R: The role of IL-1 $\beta$ in the bone loss during rheumatic diseases. Mediators Inflamm 2015: 782382, 2015.

26. Spiegelman BM: Transcriptional control of mitochondrial energy metabolism through the PGC1 coactivators. Novartis Found Symp 287: 60-63, 2007.

27. Haemmerle G, Moustafa T, Woelkart G, Büttner S, Schmidt A, van de Weijer T, Hesselink M, Jaeger D, Kienesberger PC, Zierler K, et al: ATGL-mediated fat catabolism regulates cardiac mitochondrial function via PPAR- $\alpha$ and PGC-1. Nat Med 17: 1076-1085, 2011.
28. Chen FY, Zhou J, Guo N, Ma WG, Huang X, Wang H and Yuan ZY: Curcumin retunes cholesterol transport homeostasis and inflammation response in M1 macrophage to prevent atherosclerosis. Biochem Biophys Res Commun 467: 872-878, 2015.

29. Chan KL, Pillon NJ, Sivaloganathan DM, Costford SR, Liu Z, Théret M, Chazaud B and Klip A: Palmitoleate reverses high fat-induced proinflammatory macrophage polarization via AMP-activated protein kinase (AMPK). J Biol Chem 290: 16979-16988, 2015.

30. Ho PC, Chang KC, Chuang YS and Wei LN: Cholesterol regulation of receptor-interacting protein 140 via microRNA-33 in inflammatory cytokine production. FASEB J 25: 1758-1766, 2011.

31. Choulaki C, Papadaki G, Repa A, Kampouraki E, Kambas K, Ritis K, Bertsias G, Boumpas DT and Sidiropoulos P: Enhanced activity of NLRP3 inflammasome in peripheral blood cells of patients with active rheumatoid arthritis. Arthritis Res Ther 17: 257, 2015. 